
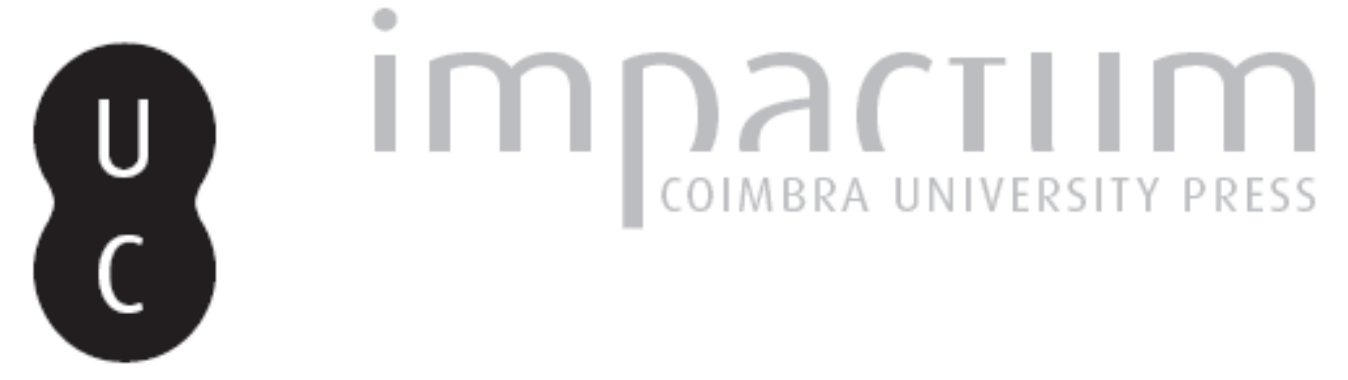

\title{
Plutarque et Euripide: quelques citations des tragédies perdues
}

\author{
Autor(es): $\quad$ Aguilar, Rosa M. ${ }^{a}$
}

Publicado por: International Plutarch Society

URL persistente:

URI:http://hdl.handle.net/10316.2/37652

DOI:

DOI:http://dx.doi.org/10.14195/0258-655X_8_1

Accessed : $\quad$ 26-Apr-2023 15:53:46

A navegação consulta e descarregamento dos títulos inseridos nas Bibliotecas Digitais UC Digitalis, UC Pombalina e UC Impactum, pressupõem a aceitação plena e sem reservas dos Termos e Condições de Uso destas Bibliotecas Digitais, disponíveis em https://digitalis.uc.pt/pt-pt/termos.

Conforme exposto nos referidos Termos e Condições de Uso, o descarregamento de títulos de acesso restrito requer uma licença válida de autorização devendo o utilizador aceder ao(s) documento(s) a partir de um endereço de IP da instituição detentora da supramencionada licença.

Ao utilizador é apenas permitido o descarregamento para uso pessoal, pelo que o emprego do(s) título(s) descarregado(s) para outro fim, designadamente comercial, carece de autorização do respetivo autor ou editor da obra.

Na medida em que todas as obras da UC Digitalis se encontram protegidas pelo Código do Direito de Autor e Direitos Conexos e demais legislação aplicável, toda a cópia, parcial ou total, deste documento, nos casos em que é legalmente admitida, deverá conter ou fazer-se acompanhar por este aviso.

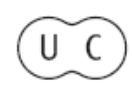




\title{
Plutarque et Euripide: quelques citations des tragédies perdues par Rosa Ma Aguilar Universidad Complutense de Madrid rmaf@filol.ucm.es
}

\begin{abstract}
We know that Plutarch likes quotations to make his opinions more significant. Our paper deals with citations of tragedies, in particular with lost tragedies of Euripides.

We aim to ascertain the number of citations in Plutarch of Euripides' lost tragedies, and then whether this number is comparable to that of the quotations from existing tragedies. Finally, we'd like also to consider the way in which he uses quotations and its suitability to his texts.
\end{abstract}

Key-Words: Plutarch, Euripides quotations, tragedy, lost tragedies.

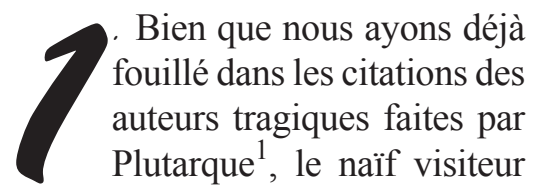

des impayables Plutach's Quotations de W. C. Hembold y E. N. O'Neil ${ }^{2}$ ne se rend pas compte de son incapacité à en faire quoi que ce soit que lorsqu'il y retourne pour la deuxième fois, tellement notre auteur aimait faire des citations en général et faire des citations des auteurs tragiques en particulier! ${ }^{3}$ Notre intention n'est pas de dire quelque chose de nouveau ou d'original, mais tout simplement d'insister sur quelques citations de pièces de théâtre perdues

1 "Plutarco y el fragmento 898 incertae fabulae de Eurípides" en Valori letterari delle Opere di Plutarco. Studi offerti al Professore Italo Gallo dall' Internacional Plutarch Society, Aurelio Pérez Jiménez \& Frances Titchener Editori, Málaga-Logan 2005, 21-28.

2 W. C.Helmbold \& E. N. O’Neill, Plutarch's Quotations, Baltimore 1959.

3 "Il predilecto" según L. Di Gregorio, "Plutarco e la tragedia greca", Prometheus (1976) 151-152, "citatto molto più di Eschilo e Sofocle messi insieme". 
pour y confirmer ce que Lamberto di Gregorio a déjà dit et étudié dans ses lucides travaux, dans Prometheus d'abord et plus tard dans Aevum. Ainsi, en réduisant graduellement les premiers objectifs, ambitieux et insensés, dans ce travail nous avons décidé de nous fixer comme but seulement les citations de incertarum fabularum que Plutarque a faites des tragédies perdues d'Euripide, et plus exactement de celles répétées en plusieurs traités, car, peut-être, et selon nous, elles constituent un document plus accord avec son côté moralisateur. Nous voudrions rechercher la source dans laquelle il a puisé et dans quelle intention elles ont été utilisées.

2. Quoique Plutarque se soit plaint du manque d'un bonne bibliothèque dans sa ville ${ }^{4}$, il ne semble pas possible qu'il ait pu extraire de ses lectures certains de ces fragments, dont plusieurs très courts, de pièce non citée par le titre - et très souvent même pas par l'auteur, seulement accompagnée d'un succinct ó $\tau \rho \alpha \gamma \omega \delta$ ós, ll semblerait plutôt qu'ils auraient fait partie de ces répertoires de "geflügelte Wörter"/mots ailés" utilisés par les auteurs cultivés pour souligner leurs jugements ou pour, en quelque sorte, les limiter. Dion Chrysostome, contemporain de Plutarque, quand il nous parle de son goût pour les auditions théâtrales, il nous apprend que des tragédies il ne restait que les passa- ges les plus résistants, nous informant ainsi que déjà à cette époque une grande partie des représentations théâtrales était formée d'anthologies de fragments remarquables, fondamentalement d'iambes, tandis que les parties correspondant au choeur avaient disparu 5 . Quand bien même cela servirait à éclairer le problème de la perte de titres de tragédies, autrefois si célèbres et populaires au point d'en préférer des anthologies des textes les plus représentatifs pour leurs idées,mis à part autres éléments de la pièce considérés de moindre intérêt en ce qui concerne le développement de la pièce-, cela ne résoudrait pas, cependant, la formation des dits minuscules fragments. Mais il semblerait possible aussi, qu'une anthologie mène à une autre et que, en passant des anthologies pour les représentations théâtrales à d'autres anthologies thématiques, les citations aient été peu à peu limitées, comme on peut voir dans le Florilegium d'Estobée. Précisément l'ample représentation d' auteurs comme Ménandre et Euripide dans cette anthologie - pensons à la préférence d'époque hélénistique par ces deux auteurs comme il arriverait par la suite aussi avec Plutarque- et en outre en ce qui concerne ce travail, la coïncidence de fragments égaux de textes chez Plutarque et Estobée fait penser à l'utilisation qu'aurait fait notre auteur d'une

\footnotetext{
4 Par exemple, dans la biographie de Démostènes (Dem. 2, 1).

5 Dio Chr. Or. 19, 5.
} 
anthologie antérieure dont l'anthologiste se serait aussi servi pour son travail, soit dans une même version soit dans une réélaboration postérieure. Ces derniers répertoires ${ }^{6}$ comporteraient des citations plus brèves composées de quelques vers ou même seulement de phrases, faites pour souligner certaines actions ou évènements avec le ton moral adéquat dont l'auteur qui s'en servirait aurait besoin à chaque occasion. Un autre indice qui témoigne de l'utilisation des dits répertoires par Plutarque est l'apparition de ces fragments dans des citations de pièces chronologiquement différentes, comme pourrait être le cas du fragment $892 \mathrm{~K}$ en De aud. poet. 36F une fois et trois dans De stoic.rep. 1043E.1044B et F, successivement.

3. Les citations de Incertarum fabularum d'Euripide chez notre auteur atteignent la somme pas négligeable de cinquante-neuf fragments - très souvent cités seulement par Plutarqueet, à notre avis représentent encore plus que d'autres citations majeures, la maintenance de vers ou de phrases, parfois très courtes, rien que pour le caractère philosophique ou sentencieux que l'auteur leur a imposé 7 . En ce qui concerne la longueur des fragments nous retrouvons diverses possibilités, des quatre vers, même si on n'en cite que deux, comme c'est le cas du fragment 899K:

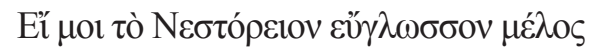

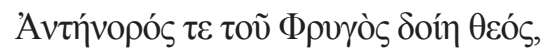

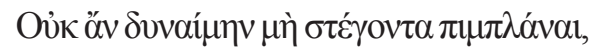

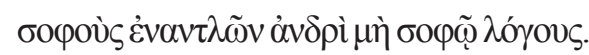
où Musgrave a reconstitué les quatre trimètres cités en général séparément en groupes de deux vers ${ }^{8}$, aux treize vers du frag. $898 \mathrm{~K}$ que Plutarque a cités dans plusieurs oeuvres, jamais avec le même nombre de vers ${ }^{9}$. Par contre, le frag. $905 \mathrm{~K}$ ne contient qu'un seul trimètre:

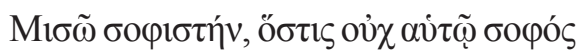
et le frag. $992 \mathrm{~K}$ ne contient que trois mots:

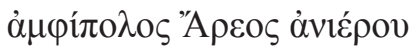

Ce que nous prétendons étudier ici ce sont à nouveau des fragments utilisés par Plutarque soit à plusieurs reprises

6 "Non dobbiamo da ultimo dimenticare che nell'antichità è esistita, già dal primo ellenismo una ricca tradizione gnomologica tramandatasi per secoli fino a Stobeo,..." en L. Di Gregorio, Aevum, 44 (1980), 78.

7 Frag. 899 Euripides, incertarum fabularum, KANNICHT. Dans les notes 1-2 et 3-4 coniunxit Musgrave.

8 Frag. 899 Euripides, incertarum fabularum, KANNICHT. En note 1-2 et 3-4 coniunxit Musgrave.

9 En De virt. mor. 442B y Amat. 756D y 770E Voir Rosa M". Agullar,"Plutarco y el fragmento 898 incertae fabulae de Eurípides", pp.24-26.

Ploutarchos, n.s., 8 (2010/2011) 3-12

ISSN 0258-655X 
dans plusieurs pièces soit à plusieurs reprises dans la même pièce, afin de voir comment ce mécanisme de citations a pu être originé.

3.1. Nous commencerons par les fragments qui ont été cités à quatre occasions.

Le premier, si l'on suit le numéro du fragment dans l'édition de Kannicht est le fragment 892, cité plus haut:

De aud.poet. 36.F:

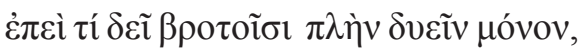

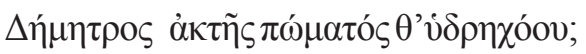

parce que, de quoi les mortels ont-ils besoin si ce n'est que de deux choses:

le blé de Déméter et la boisson d'eau pure? ${ }^{10}$

Ce fragment comporte trois vers mais Plutarque n'en a cité que les deux premiers. Et il en est ainsi car il cherche à ce que le jeune étudiant soit capable de mieux concilier les expériences contradictoires de la vie quotidienne dans son étude des philosophes une fois qu'il aura assimilé la sagesse des poètes en écoutant et en lisant des vers comme ceux-là.

Dans De stoic. rep. 1043E notre auteur cite à nouveau ce même fragment et également les deux premiers vers. Dans ce cas il s'agit de sa critique à
Chrisippe, qui considère que le sage doit pouvoir s'enrichir grâce à ses connaissances et il fait mention de trois domaines dont il peut tirer son profit, la royauté, les amis et l'exercice de la sophistique. Le brave Plutarque ne semble pas être spécialement scandalisé par ces sources de richesse, mais plutôt par les contradictions du stoïque, puisque fréquemment, "dans beaucoup de passages il fait un éloge exténuant de ces vers" et il les fait ainsi apparaître après $^{11}$. En effet, il est quelque peu choquant la conciliation entre le gain, même si la façon dont il est obtenu est honnête, et l'éloge à outrance du pain et de l'eau comme seuls besoins de l'homme, si peu sage soit-il. En 1044B il revient à nouveau sur ces deux vers pour illustrer les contradictions de Chrisippe. Celui-ci, dans son traité Sur la République disait que les citoyens ne devaient rien se procurer par plaisir et il louait les deux célèbres vers, dont Plutarque dans cette occasion mentionne le nom de l'auteur. Mais le stoïque,en flagrante contradiction, louait aussi Diogènes pour s'être masturbé publiquement et pour avoir désiré en finir avec la faim en se frottant l'estomac. Et dans ce cas, Plutarque s'indigne beaucoup plus d'une pareille effronterie de la part du cynique et de l'éloge du stoïque. Dernièrement, en

10 Trad. de J. García LóPeZ, Obras morales y de costumbres I, Madrid 1985, pp. 157-158.

11 Trad. de Raúl Caballero, Obras morales y de costumbres XI, Madrid 2004, pp. 268-269. 
1044F il fait réellement une adaptation du deuxième vers ${ }^{12}$.

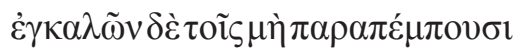

$\tau \alpha \tilde{\tau} \tau \alpha \mu \eta \delta$ '

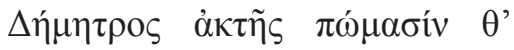

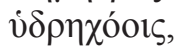

mais il dénonce ceux qui ne méprisent pas ces choses et à qui le grain de Déméter et la liquide boisson d'aqueux fluer ne suffisent pas... ${ }^{13}$

Dans cette citation la finalité est aussi celle de souligner les contradictions de Chrisippe dans ses critiques aux plaisirs qui nous sont fournis para la vue et l'ouie, en introduisant une similitude où figure l'adaptation.

Connaissant le penchant des stoïques pour la citation de vers ${ }^{14}$ il n'aurait rien d'étonnant que l'utilisation du frag. 892 dans Deaud. poet.36F ne vienne pas d'un répertoire mais précisément des lectures que Plutarque à coup sûr aurait faites de pas mal d'oeuvres de Chrisippe. Même si les De stoicorum repugnantiis sont sans doute une oeuvre postérieure a $D e$ audiendis poetis la chronologie relative pour cette hypothèse ne représente pas un obstacle puisque Plutarque aurait pu lire Chrisippe avant la composition de ce dernier traité et même pendant sa jeunesse. D'un autre côté, on pourrait nous objecter qu'on parle de quatre citations alors que seulement trois appartiennent à une seule oeuvre, mais cela se justifie si l'on pense qu'il s'agit de répétitions de la même citation dans des contextes différents et avec une finalité aussi différente.

Même s'il ne cite pas l'auteur, pour ce que nous venons de dire Plutarque aurait pu le connaître à travers ses lectures de Chrisippe.

3.2. Le deuxième des fragments cités à quatre occasions par Plutarque est le 1086K:

De adulatore et amico, 71E:

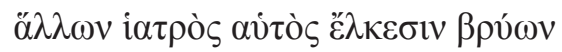

Tout en étant couvert de plaies tu fais le médecin des autres ${ }^{15}$.

Dans ce traité bourré de citations, pour souligner sa pensée, notre auteur utilise ce trimètre opportunément et sans l'encercler par d'autres citations n'ayant pas trop de sens, comme il fait souvent. Plutarque prévient qu'un homme ne possédant pas de grandes vertus, avant de réprimander quelqu'un avec franchisse doit écouter la phrase en question au cas où lui aussi serait concerné par ce dont elle prévient. Il n'y a pas d'allusion à son auteur.

12 Comme dit Kannicht en ce qui concerne cette citation: 2-3 Plut. 11p- 1044F verbis contextui Plutarchi accommodatis.

13 Trad. de Raúl CABallero, Obras morales y de costumbres XI, Madrid 2004, p. 273.

14 “....in spezial modo presso gli Stoici, di introdurri a pieni mani nel discorso citazioni in 
De capienda ex inimicis utilitate 88D:

Ici la citation apparaît parmi d'autres de tragédie et de poésie (Eschyle, Pindare) et de prose (Démosthène), et notre auteur conseille ensuite, de s'examiner soi-même si on va blâmer les autres, ce qui vient apostillé par ce trimètre. L'emploi est juste et la mention d'auteur est celle de $\tau$ ò $\tau o \tilde{v} \tau \rho \alpha \gamma \omega \delta$ oṽ,sans plus de précisions.

\section{De fraterno amore 481A:}

Dans ce passage l'utilisation de la citation est cohérent et conforme à la doctrine enseignée:

Qui a vieilli en procès, discussions et jugements contre ses frères, en conseillant après à ses fils la concorde,médecin des autres, alors qu'il est lui-même couvert de plaies, affaiblit son raisonnement avec ses œuvres ${ }^{16}$.

Par contre, cette citation appartient bien un peu au groupe des citations assemblées, parce que Plutarque ajoute ensuite deux autres des Phoeniciennes d'Euripide pour insister sur la condui- te inopportune entre frères, en nous présentant Etheoclès dans des harangues contradictoires d'abord envers ses enfants, ensuite envers son frère et un peu plus loin envers Atrée de façon similaire $^{17}$.

\section{Adversus Colotem 1110E:}

Après avoir censuré Colotes à son maître de telle façon qu'il l'enlise avec lui dans son relativisme, selon une catégorique affirmation de Plutarque à la fin du c.7, le suivant commence par la question si l'épicurien ne sera pas "médecin des autres alors qu'il est lui-même couvert de plaies"18, pour y répondre de manière également rhétoricienne qu'il n'en est nullement ainsi, que dans ses censures à Démocrite il enlève aussi la vie à son maître, Épicure $^{19}$, en renforçant maintenant cette affirmation avec d'autres citations de Démocrite. L'emploi du vers dans ce cas nous semble en effet quelque peu forcé d'autant plus qu'il est placé en tête du texte, et qu'il est prédicat de la phrase ${ }^{20}$.

versi di ogni genere," en L. DI Gregorio, Aevum, 53 (1979) 16.

15 Trad. de J. García López, Obras morales y de costumbres I, Madrid 1985, pp. 259 y 313.

16 Trad. de Rosa Ma. Aguilar, Obras morales y de costumbres VII, Madrid 1995, p. 168.

17 Phoeniciennes 504-506;536-38 -mais ces derniers vers c'est Yocaste qui les dit et pas Etheoclès- et $\mathrm{NAUCK}^{2}$, adesp. 384.

18 Trad. de Francisco Martos Montiel, Obras morales y de costumbres XII, Madrid 2004, p.60.

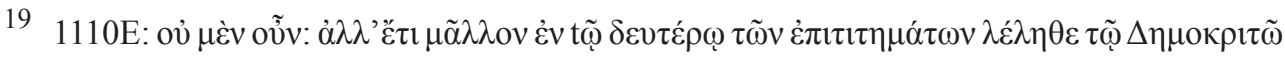

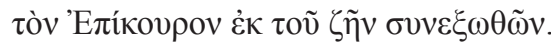

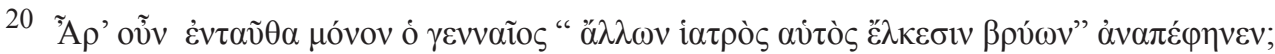


Ce vers semble avoir joui d'une certaine célébrité dans l'antiquité. Il est cité par Galène et il apparaît aussi dans Grégoire de Nazianzos et dans d'autres auteurs chrétiens. Comme nous avons déjà vu, Plutarque ne mentionne pas Euripide comme étant son auteur et seulement dans De cap. il mentionne "l'auteur tragique". Il semblerait être la Suda où se trouve l'attribution à Euripide ${ }^{21}$.

4. Fragments utilisés à trois occasions:

\subsection{Fragment $941 \mathrm{~K}$}

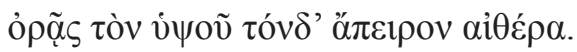

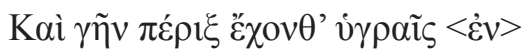
$\dot{\alpha} \gamma \kappa \alpha ́ \lambda \alpha 1 s$,

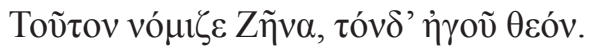

Ce fragment est composé de trois vers, mais chez notre auteur la citation n'apparaît jamais complète:

De exilio, 601 A ne montre que les deux premiers:

Vois-tu dans les hauteurs cet éther infini

Qui soutient dans son entourage la terre dans une humide étreinte?

Avant la citation, Plutarque rappelle que Socrates ne se considérait ni athénien ni hélène, mais cosmios, c'est à dire, habitant de l'univers et il utilise les vers d'Euripide pour conseiller au déterré de considérer ainsi les frontières de la patrie et pas les étroites limites des pays. La citation résulte donc cohérente et il est compréhensible que le troisième trimètre ait été supprimé.

\section{Ad principem ineruditum $780 \mathrm{D}$ :}

Dans ce traité de politique, la citation ne se fait que des deux premiers vers également, mais ici elle semble un peu forcée. Plutarque utilise ces vers comme déclencheurs de son argumentation plutôt que comme soutien à sa pensée. Dans un contexte plein d'allusions à la nature et qui montre des similitudes avec d'autres passages d'Eschyle et d'Euripide lui-même ${ }^{22}$, la conclusion assez forcée est que tous ces biens offerts par la nature ne sont possibles que s'il existe la loi, la justice et un bon gouvernement. Les vers ne sont donc qu'un simple appui rhétorique.

\section{Aetia physica 919B:}

On y cite seulement les deux premiers vers. Dans une question qui, dans son commencement, se pose sur la température des eaux se demandant si le froid est le manque de chaleur, on introduit de façon forcée les vers pour assurer que beaucoup de gens n'admirent pas la beauté des phénomènes qu'on y décrit.

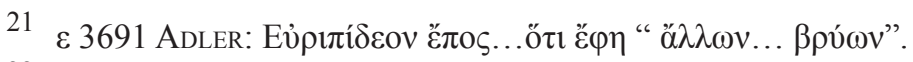

22 Eschyle, frag. 44 RADT; Euripides, frag. 898 KANNICHT. A propos de ces fragments voir RosA Ma . Aguilar, "Plutarco y el fragmento 898 incertae fabulae de Eurípides", pp. 21-22. 
5. Le reste des citations de incertarum fabularum apparaissent désormais dans deux traités seulement, c'est à dire dans les frags 895, 905, 958, 961, 970, 971, 972, 973, 974, 989, 994, 996 y 980 Kannicht ou bien elles apparaissent dans une seule oeuvre. Les traiter toutes se révèle maintenant une tâche impossible. Cependant, le 980K apparaît à deux reprises, mais dans le même traité, De sera numinis vindicta, 556E, 562E. Nous conclurons avec lui notre travail à la recherche d'une nouvelle perspective ${ }^{23}$.

Fragment 980K:

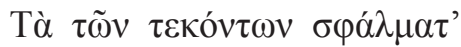

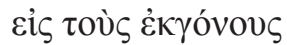

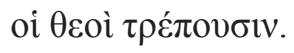

De sera numinis vindicta 556E, 562E:

Les pêchés des parents sur les enfants

Ce sont les dieux qui les envoient $^{24}$.

Dans la première occasion, Plutarque mentionne Euripide qui reproche aux dieux la doctrine de la faute héré-

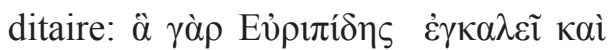

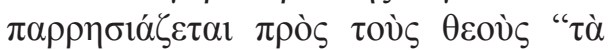

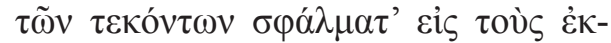

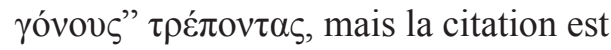
seulement du premier vers et elle est adaptée. Cependant, la deuxième fois il inclue les deux vers dans son discours et sans mentionner maintenant l'auteur tragique, il argumente que :

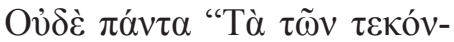

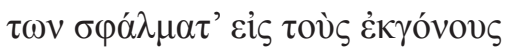

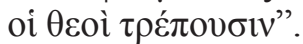

Même pas tous les pêchés des parents sur les enfants sont envoyés par les dieux.

De cette façon, la première citation constitue un argument de l'auteur tragique contre la doctrine traditionnelle, incluse par Timon dans son raisonnement, tandis que la négation qui précède la deuxième citation sert à dire le contraire.

Cette utilisation d'un même texte à deux fins différentes semble faire preuve de la versatilité de notre auteur dans le maniement de citations, et peut être aussi de son inconsistance.

A partir des fragments étudiés et de leur insertion dans le texte nous pouvons faire quelques considérations sur la

23 Nous nous sommes occupés de préférence des citations de incertarum fabularum de Euripide entre autres raisons parce que L. DI GREGORIO s'était déjà occupé des citations de fragments de pièces perdues mais avec titre et aussi de celles transmises dans Aevum, 53 (1979) 46-79. Il y a une thèse de C. S. J. Mitchell, An Analysis of Plutarch's Quotations from Euripides, Diss. Univ. of Southern California, Los Angeles 1968 (microf.), dont nous avons entendu parler par L. Di Gregorio, Aevum, 53 (1979) 11, n. 2 et passim, mais à laquelle nous n'avons pas eu accès.

24 Trad. de Rosa Ma. Aguilar, Obras morales y de costumbres VIII, Madrid 1996, pp. 138 y 153. 
consistance de la citation et surtout de l'emploi qu'en fait Plutarque.

Des citations cohérentes avec la pensée exposée venant la renforcer, comme dans De aud. Poet. 36F, De exilio 601 A, De adulatore et amico 71E.

Des citations cohérentes avec la pensée exposée, mais un peu forcées et bourrées parmi bien d'autres, comme De capienda ex inimicis utilitate 88D ou De fraterno amore $481 \mathrm{~A}$.

Des citations excessivement forcées dans le texte, comme Adversus Colotem 1110E.

Des citations servant à souligner l'incohérence de la pensée d'un autre, comme De stoicorum repugantiiis 1043E.

Des citations servant à entamer une argumentation, comme Ad principem ineruditum $780 \mathrm{D}$, encore que nous la trouvions aussi quelque peu forcée.

Comme nous avons pu voir, la casuistique est très variée. Aussi, en conclusion, continuons-nous de penser que les citations de Plutarque relèvent plus de parure et d'ornement du texte que de véritables réflexions pour mieux expliquer et argumenter ses raisonnements. En somme, elles ne représenteraient, à notre avis, qu'une conséquence de son goût pour la poésie dans une application de la rhétorique.

\section{RÉFÉRENCES BIBLIOGRAPHIQUES}

Aguilar, Rosa $\mathrm{M}^{\mathrm{a}}$,

- "Plutarco y el fragmento 898 incertae fabulae de Eurípides" en A. PÉREZ JIMÉNEZ \& F. Titchener (Eds.) 2005, pp. 21-28.

Di Gregorio, L.,

- "Plutarco e la tragedia greca", Prometheus, 2 (1976) 151-174.

- "Lectura diretta e utilizzazione di fonti intermedie nelle citazioni plutarchee dei tre grande tragici I", Aevum, 53 (1979) 11-50.

- "Lectura diretta e utilizzazione di fonti intermedie nelle citazioni plutarchee dei tre grande tragici II", Aevum, 54 (1980) 46-79.

Helmbold, W.C. \& O’Neill, E. N.,

- Plutarch's Quotations, Baltimore, 1959.

Hense, O.,

- "Iohannes Stobaios", RE, cols. 25492586. s.v.

KANNICHT, R.,

- Tragicorum graecorum fragmenta V , Euripides (2 vols), Göttingen, 2004.

Pérez Jiménez, A. \& Titchener, F. (Eds.),

- Valori letterari delle Opere di Plutarco. Studi offerti al Professore Italo Gallo dall' Internacional Plutarch Society, Málaga-Logan, 2005.

TAgLiasacchi, A. M.,

- (1960) "Plutarco e la tragedia greca", Dioniso, (1960) 124-142. 